\title{
(6) OPEN ACCESS \\ Neonatal outcomes following new reimbursement limitations on palivizumab in Italy
}

\author{
Valeria Belleudi, Francesco Trotta, Luigi Pinnarelli, Marina Davoli, Antonio Addis
}

Department of Epidemiology, Lazio Regional Health Service, Roma, Italy

\section{Correspondence to}

Dr Antonio Addis, Department of Epidemiology, Lazio Regional Health Service, Roma 00147 Italy; agmaddis@gmail.com

Received 16 April 2018 Revised 19 July 2018 Accepted 24 August 2018 Published Online First 14 September 2018
Check for updates

(C) Author(s) (or their employer(s)) 2018. Re-use permitted under CC BY-NC. No commercial re-use. See rights and permissions. Published by BMJ.

To cite: Belleudi V, Trotta F, Pinnarelli L, et al.

Arch Dis Child

2018:103:1163-1167.

\section{ABSTRACT \\ Objective To evaluate the impact of new} reimbursement decisions for palivizumab treatment on respiratory syncytial virus (RSV) hospitalisations and the concomitant number of palivizumab prescriptions for infants aged $<2$ years.

Design We compared the RSV hospitalisation rates in infants before and after implementation of new limitations during three RSV seasons 2014-2017.

Setting Population aged $<2$ years at the beginning of each RSV seasons extracted from regional health systems (Lazio region, 2016, 5898124 inhabitants and 47595 births).

Patients Out of 70323 infants, 5895 (8.4\%) premature babies (gestational age $(G A)<37$ weeks) were followed before-after Italian Medicines Agency (AIFA)-2016 limitations.

Intervention In 2016, AIFA, following the American Academy of Pediatrics guidelines, decided to limit coverage of palivizumab prophylaxis (GA $\leq 29$ weeks). Main outcomes measures Trend of hospitalisations by months and rate of RSV before-after new restrictions were analysed. Palivizumab prescriptions and costs for National Health Service (NHS) were considered.

Results In a population of 284902 aged $<2$ years, the number of hospitalisations due to RSV infection was 1729. Following AIFA-2016 limitations, a reduction in the number of RSV infection-based hospitalisations from $6.3 / 1000(95 \% \mathrm{Cl} 6.0$ to 6.7$)$ to $5.5 / 1000(95 \% \mathrm{Cl} 5.0$ to 5.9) was observed. Palivizumab showed a concomitant reduction of $48 \%$ in the number of prescriptions (saving $€ 750000$ for the NHS). No differences of GA, age on admission or severity of RSV infection were observed. Conclusions Implementation of the new palivizumab reimbursement criteria was not associated with an increase in the RSV hospitalisation rate for children aged $<2$ years despite a significant reduction in the number of palivizumab prescriptions.

\section{INTRODUCTION}

The respiratory syncytial virus (RSV) is considered worldwide to be the most common cause of infection of the respiratory tract in children aged $<2$ years and a major public health burden associated with frequent hospitalisation of children. ${ }^{1}$ Several studies defining the epidemiology and burden of the RSV among infants have shown that, in Italy, the RSV is associated with $31 \%-49 \%$ of all acute respiratory infections. ${ }^{2-7}$ The risk of hospitalisation decreases during the first 2 years of life but has been reported to be higher according to the grade of prematurity, and in infants with chronic lung disease or other congenital diseases. ${ }^{8}$

\section{What is already known on this topic?}

Palivizumab can reduce the prevalence of respiratory syncytial virus infection-based hospitalisation among premature infants in clinical trials and is particularly recommended for high-risk populations.

- The best target among such populations is controversial.

\section{What this study adds?}

- Restricted use of palivizumab in infants aged $<2$ years following reimbursement limitations by a national regulatory authority in Italy was not associated with an increase in hospitalisation prevalence despite a significant reduction in the number of prescriptions in the same target population.

Palivizumab is a humanised murine monoclonal antibody and it is the main pharmacological agent used for the prevention of RSV infections. Following the Impact-RSV study, the drug has been approved in the USA and Europe based on data showing a significant reduction in hospitalisations for RSV disease in premature infants and in infants with chronic lung disease after monthly intramuscular administration of palivizumab throughout RSV season. ${ }^{9}$

Since the introduction of palivizumab onto the market, palivizumab has been associated with an intense debate regarding the best target populations where to be used. Such controversy has resulted from conflicting data on efficacy in different risk categories and due to its expense. In this context, several recommendations for its administration have been published. ${ }^{10} 11$

The therapeutic indication reported in the Summaries of Product Characteristics (SPC) approved by the European Medicine Agency states that palivizumab can be used for the prevention of severe disease in the lower respiratory tract necessitating hospitalisation caused by the RSV in children at high risk for RSV disease. High-risk categories were identified in children: (i) born at $\leq 35$ weeks of gestation and $<6$ months of age at the onset of the RSV season; (ii) $<2$ years of age and requiring treatment for bronchopulmonary dysplasia within the previous 6 months; (iii) $<2$ years of age and with haemodynamically significant congenital heart disease. 
In Italy, following publication of the revised recommendations from the American Academy of Pediatrics (AAP) in 2014, ${ }^{10}$ prescribers and decision makers tried to define a new strategy for the identification of paediatric patients for whom prophylaxis with palivizumab is more effective. The review of all data on efficacy and safety convinced the Italian Medicine Agency (AIFA) to adopt new limitations for palivizumab in terms of setting criteria for reimbursements by the National Health Service (NHS). Starting October 2016, palivizumab was reimbursed only for children: (i) born at $\leq 29$ weeks of gestation; (ii) $<2$ years of age and requiring treatment for bronchopulmonary dysplasia within the previous 6 months; (iii) $<1$ year of age and with haemodynamically significant congenital heart disease. Furthermore, even if not included in the SPC of the drug, AIFA recommended reimbursement by the NHS also for prophylaxis with palivizumab for children: (iv) $\leq 1$ year of age and with severe congenital malformations (eg, neuromuscular, cardiac); (v) $\leq 2$ years of age in children with primitive or secondary immunodeficiencies.

New limitations for reimbursements were more compliant with the AAP-2014 recommendations and restricted the potential use of palivizumab when comparing the eligible neonatal population with those included in the official approved therapeutic indication reported in the SPC.

After considerable discussion among the Ministry of Health, the Italian Society of Neonatology and patient associations, in October 2017 AIFA decided to revoke the new limitations eliminating the reimbursement restrictions for infants born at gestational age (GA) 30 to $\leq 35$ weeks.

We wished to ascertain, using real-world data, the impact of the reimbursement limitations during 2016-2017 for prophylaxis with palivizumab in a paediatric population in terms of hospitalisation prevalence and drug consumption.

\section{METHODS}

We compared the prevalence of RSV infection-based hospitalisations in patients aged $<2$ years before and after implementation of the 2016 limitations for the reimbursement of palivizumab by the Italian NHS.

Data were extracted from three main sources. The first source was the Certificate of Delivery Care (CEDAP up to 2016). This reports information at birth, such as GA, birth weight, sex and date of birth. It also reports information on the health status and sociodemographic characteristics of the mother.

The second source was the Drug Claims Registry. This collects information on drug prescriptions reimbursed by the healthcare system and dispensed by private or public pharmacies or, in some cases, directly from local health units or hospital pharmacies. For each prescription, the following information is available: patient code, date of prescription, drug substance, marketing authorisation code and number of packages. All drugs are identified through the international Anatomical Therapeutic Chemical (ATC) classification system.

The final data source was the Hospital Information System. This collects information on all hospital discharges registered in a regional hospital, in particular the dates of admission and discharge, diagnoses and procedures (primary and secondary) according to the International Classification of Diseases, Ninth Revision, Clinical Modification (ICD-9-CM) and internal transfer (such as admission to an intensive care unit).

Three RSV seasons (from 1 October to 30 April) were defined: two before (2014-2015; 2015-2016) and one after (2016-2017) the new AIFA reimbursement limitations.

To identify the target population, all infants born during 2012-2016, discharged alive and resident in the Lazio region,

Table 1 Population at risk of respiratory syncytial virus infection (infants $<2$ years of age) and hospitalisation before and after AIFA-2016 limitations

\begin{tabular}{|c|c|c|c|c|c|c|}
\hline Population at risk at the start of the RSV season & \multicolumn{2}{|c|}{$\begin{array}{l}\text { Before AIFA-2016 limitations } \\
\text { RSV seasons 2014-2016 }\end{array}$} & \multicolumn{2}{|c|}{$\begin{array}{l}\text { After AIFA-2016 limitations } \\
\text { RSV season } 2017\end{array}$} & \multicolumn{2}{|l|}{ Total } \\
\hline Children $<24$ months & 193386 & & 91516 & & 284902 & \\
\hline Children $<6$ months & 47608 & & 22715 & & 70323 & \\
\hline Premature baby ( $<37$ weeks of gestation) & 4069 & $8.5 \%$ & 1826 & $8.0 \%$ & 5895 & $8.4 \%$ \\
\hline \multicolumn{7}{|l|}{ Gestational age at birth* } \\
\hline$>35$ and $<37$ & 1714 & $3.6 \%$ & 757 & $3.3 \%$ & 2471 & $3.5 \%$ \\
\hline$>32$ and $\leq 35$ & 1714 & $3.6 \%$ & 765 & $3.4 \%$ & 2479 & $3.5 \%$ \\
\hline$>29$ and $\leq 32$ & 444 & $0.9 \%$ & 191 & $0.8 \%$ & 635 & $0.9 \%$ \\
\hline$\leq 29$ & 197 & $0.4 \%$ & 113 & $0.5 \%$ & 310 & $0.4 \%$ \\
\hline \multicolumn{7}{|l|}{ Hospitalisations } \\
\hline All causes & 28549 & & 12614 & & 41163 & \\
\hline Respiratory virus & 3508 & & 1620 & & 5128 & \\
\hline RSV & 1228 & & 501 & & 1729 & \\
\hline \multicolumn{7}{|l|}{ Age (months)* } \\
\hline 0 & 242 & $19.7 \%$ & 108 & $21.6 \%$ & 350 & $20.2 \%$ \\
\hline 1 & 339 & $27.6 \%$ & 126 & $25.1 \%$ & 465 & $26.9 \%$ \\
\hline 2 & 220 & $17.9 \%$ & 90 & $18.0 \%$ & 310 & $17.9 \%$ \\
\hline 3 & 116 & $9.4 \%$ & 53 & $10.6 \%$ & 169 & $9.8 \%$ \\
\hline 4 & 63 & $5.1 \%$ & 39 & $7.8 \%$ & 102 & $5.9 \%$ \\
\hline 5 & 51 & $4.2 \%$ & 20 & $4.0 \%$ & 71 & $4.1 \%$ \\
\hline 6 & 40 & $3.3 \%$ & 12 & $2.4 \%$ & 52 & $3.0 \%$ \\
\hline$>6$ & 157 & $12.8 \%$ & 53 & $10.6 \%$ & 210 & $12.1 \%$ \\
\hline
\end{tabular}

${ }^{*} P>0.05,0.1807$ and 0.2951 , respectively $\left(\chi^{2}\right.$ test).

AIFA, Italian Medicines Agency; RSV, respiratory syncytial virus. 


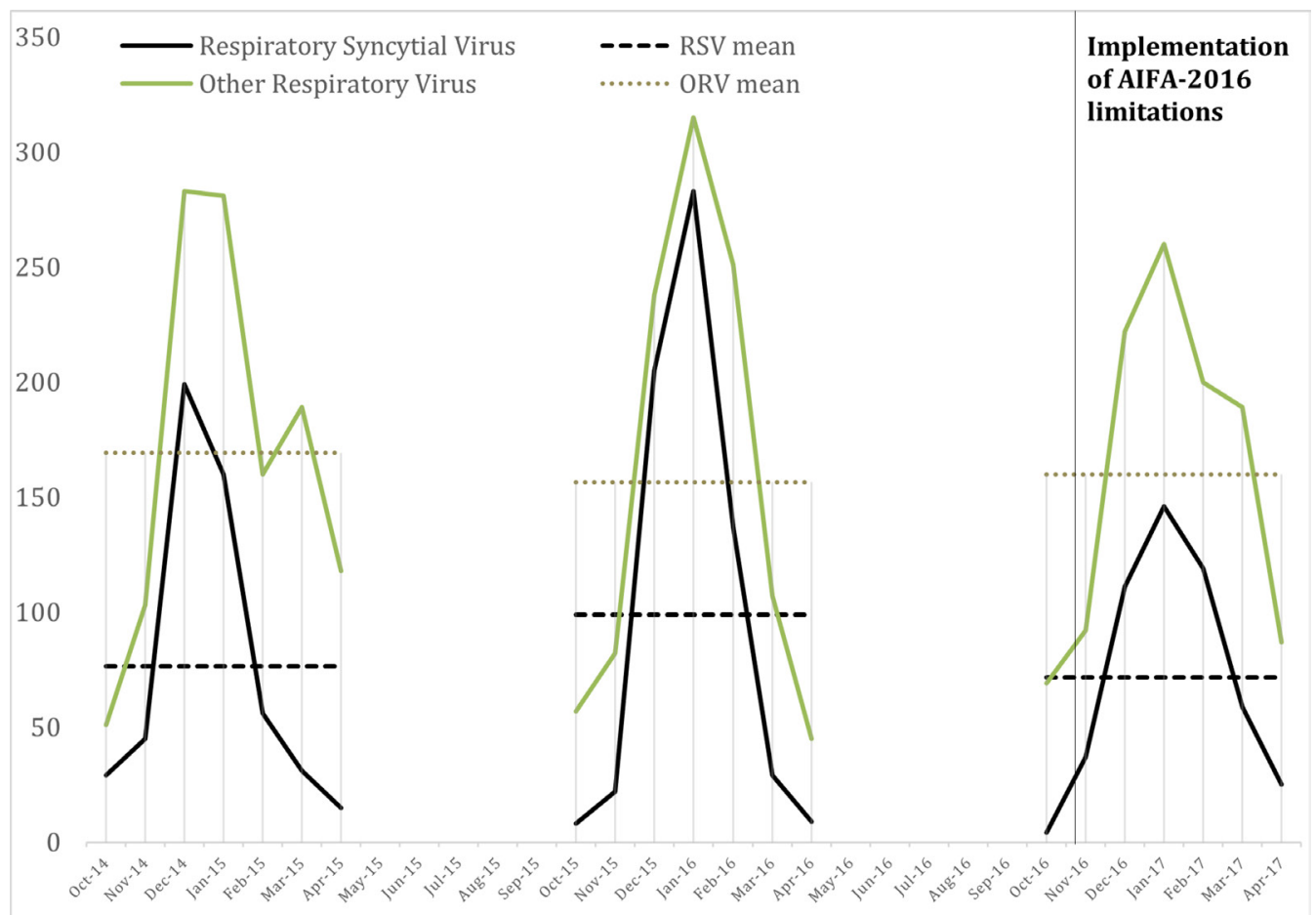

Figure 1 Hospitalisations for infection by the respiratory syncytial virus (RSV) and other respiratory viruses (ORV) before and after Italian Medicines Agency (AIFA)-2016 limitations.

were extracted by CEDAP. For each RSV season, infants aged $<2$ years at the beginning of the season were considered.

During the three seasons, hospitalisations for infants aged $<2$ years for all causes, with a diagnosis of RSV infection (ICD-9-CM $079.6 ; 466.11 ; 480.1)$ or with a diagnosis of other respiratory virus (ORV) infection (ICD-9-CM 466.19; 480 excluding 480.1) were extracted. RSV hospitalisation necessitating oxygen therapy or mechanical ventilation (ICD-9-CM 93.90; 96.7) was also identified.

In the same periods, palivizumab prescriptions (ATC J06BB16) for infants aged $<2$ years were documented.

A descriptive analysis of the population at risk and hospitalisations was presented. A before-and-after comparison between GA classes for the target population and between age at the time of admission for hospitalised infants was done by the $\chi^{2}$ test. The trend for hospitalisations due to infection by the RSV and ORV by month during each RSV season was described.

The prevalence of RSV infection before and after the new AIFA reimbursement limitations and their CIs were calculated assuming a Poisson distribution. The prevalence of oxygen therapy and mechanical ventilation in hospitalised RSV-infected infants was also evaluated. Finally, the impact of new reimbursement criteria (2016-2017) on palivizumab prescriptions was considered.

\section{RESULTS}

Of 284902 infants aged $<2$ years identified in the databases of regional health services during the three seasons of epidemic RSV infection, 70323 were aged $<6$ months (table 1). Of these, 5895 (8.4\%) were premature babies (GA <37 weeks). A significant difference in GA before and after new reimbursement criteria was not observed $(\mathrm{p}=0.1807)$.

During the three RSV-infection seasons, 2014-2017, >41000 hospitalisations for infants aged $<2$ years were recorded. Of these, $12.5 \%(n=5128)$ were regarded to be hospitalisations due to infection by a respiratory virus, with 1729 cases of RSV infection-based hospitalisation. The distribution of age on admission for RSV infection-based hospitalisation was not significantly different before the new AIFA reimbursement limitations $(\mathrm{p}=0.2951)$.

Figure 1 shows the trend for infection by the RSV and ORVs according to the three seasons (1 October 2014 to 30 April 2015; 1 October 2015 to 30 April 2016; 1 October 2016 to 30 April 2017). Following the introduction of the new AIFA reimbursement limitations, an increase in the number of hospitalisations due to respiratory-virus infections was not observed. In particular, monthly averages for the number of RSV infection-based hospitalisations for each of the three seasons were 76, 99 and 72, respectively. This corresponded to a reduction of

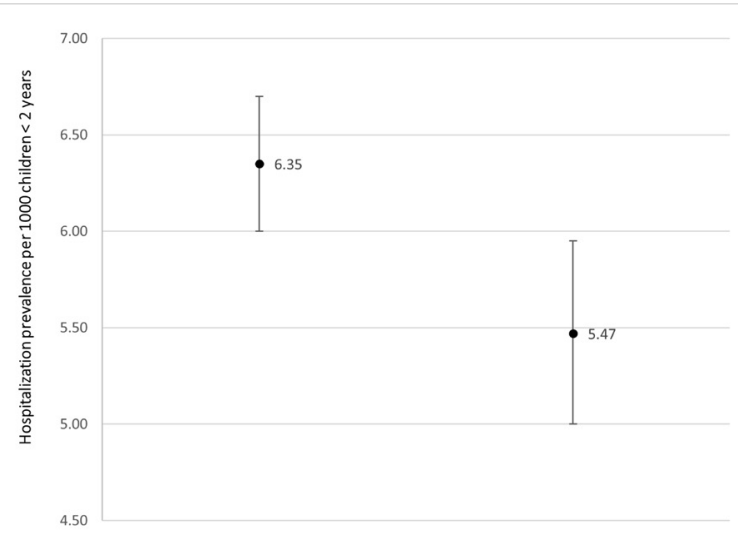

Figure 2 Prevalence of infection by the respiratory syncytial virus before and after Italian Medicines Agency-2016 limitations. 


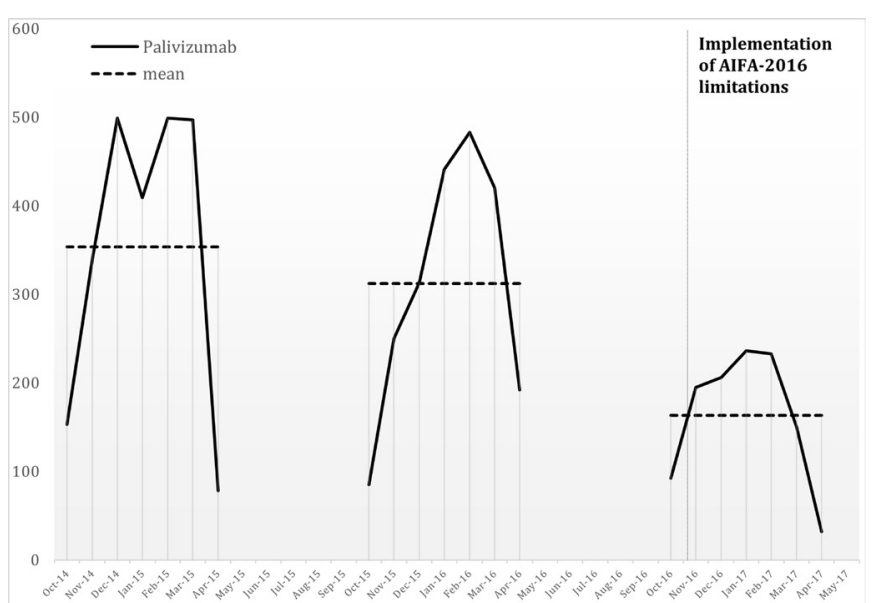

Figure 3 Number of palivizumab prescriptions before and after Italian Medicines Agency (AIFA)-2016 limitations.

RSV infection-based hospitalisations before and after the AIFA2016-2017 reimbursement limitations of 18\%.

Figure 2 shows a reduction in the prevalence of RSV infection-based hospitalisation before and after the reimbursement criteria set by the NHS in 2016. In particular, the prevalence of hospitalisation in infants aged $<2$ years in the RSV seasons 2014-2016 vs 2016-2017 was 6.3/1000 (95\% CI 6.0 to 6.7) and $5.5 / 1000$ (95\% CI 5.0 to 5.9), respectively. Furthermore, the prevalence of oxygen therapy and mechanical ventilation in RSV-infected infants hospitalised was similar in both seasonal periods (9.6\% vs $9.2 \%$; $=0.7832$; data not shown).

Figure 3 shows the trends of palivizumab prescriptions before and after implementation of the new reimbursement limitations. In the period following the final regulatory decision, a reduction in the number of palivizumab doses reimbursed by the regional NHS of $48 \%$ compared with previous seasons was noted. This reduction was associated with an estimated saving for $€ 750000$ by the NHS.

\section{DISCUSSION}

Our study provides data on the impact of the reimbursement decision by the Italian NHS based on international guidelines. ${ }^{10} 11$ A significant reduction in the prevalence of palivizumab administration between the pre-2016 and post-2016 reimbursement decision was not associated with an increase in the prevalence of RSV infection-based hospitalisation for children aged $<2$ years. In fact, we detected a reduction in prevalence, which could be plausible with more accurate coding by hospital administrations linked with the new reimbursement procedures or with an increase of palivizumab compliance in babies with a higher risk of RSV infection. Another possible explanation could be the year-to-year variation in the intensity of RSV epidemics. However, in our study, the prevalence of RSV-based hospitalisation (6.3 and 5.5 per 1000 children aged $<2$ years) and the epidemiological characteristics of our populations were not different to those reported by Grindeland et al in the USA. ${ }^{12}$ In their retrospective before-and-after analysis, they observed a prevalence of hospitalisation of 5.37 per 1000 children aged $<2$ years in the pre-2014 guideline period vs 5.78 in the post- 2014 guideline period $(\mathrm{p}=0.622)$. In accordance with our conclusions, the authors suggested that implementation of the 2014 guideline was not associated with an increase in the prevalence of RSV infection-based hospitalisations for children aged $<2$ years with a significant concomitant lower use of palivizumab. ${ }^{12}$
We also took into account hospitalisations related to ORV infections, and no differences in the epidemic seasons were detected. Instead, in the same population, we observed a significant reduction in the number of palivizumab prescriptions. Application of the data from our study population revealed that, in terms of the reduction of prevalence of palivizumab prescriptions $(-48 \%)$, up to the national level (473 438 newborns in Italy in 2016), we estimated a nationwide saving of $€ 7.5$ million in the first year following the 2016-2017 reimbursement limitations.

Other studies support our results showing no differences in the prevalence of RSV infection-based hospitalisations in children aged $<2$ years after implementation of the AAP-2014 guidance for palivizumab use. ${ }^{1314}$ In particular, Rajah et al documented the difference in the number of RSV infection-based hospitalisations and other neonatal outcomes in infants aged $<1$ year with a GA of 29-34 weeks in the seasons before and after implementation of the AAP-revised guidance. In that retrospective study, RSV infection was confirmed by virology results and, of 1063 RSV infection-based hospitalisations in infants aged $<1$ year, $7.1 \%$ vs $9.8 \%$ $(p=0.1)$ were observed before and after the AAP-2014 guideline, respectively. Conversely, this study observed an increase in disease severity in premature infants aged $<6$ months born at a GA of 29-34 weeks who were no longer eligible for palivizumab prophylaxis. However, similar to our data, palivizumab eligibility decreased to $32.3 \% .^{13}$

During three consecutive seasons from 2012 to 2014, Farber et al provided additional support to the AAP-2014 guidelines for palivizumab prophylaxis. ${ }^{14}$ They evaluated the prevalence of RSV infection-based hospitalisation in 2031 infants of GA 29-32 weeks and 12066 infants of GA 33-36 weeks. Differences in the prevalence of RSV infection-based hospitalisations in infants of GA 29-32 weeks receiving palivizumab compared with those who did not receive prophylaxis was reported $(3.1 \%$ vs $5.0 \%, \mathrm{p}=0.04)$. However, a difference in the prevalence of RSV infection-based hospitalisations in infants of GA 33-36 weeks was not observed regardless of palivizumab receipt. Interestingly, palivizumab prophylaxis was associated in infants of GA 29-32 weeks with a higher prevalence of hospitalisation for bronchiolitis because of ORVs.

Evidence of lack of efficacy of palivizumab in infants aged $<2$ years born at a GA of 29-35 weeks without comorbidities has been supported by several studies. ${ }^{15-17}$ That is, no significant difference in the prevalence of RSV infection-based hospitalisation in those treated with or without palivizumab has been documented.

A small, retrospective single-centre study in Italy investigated the impact of the AAP-2014 guideline on the prevalence of RSV infection-based hospitalisation in infants aged $\leq 1$ year. ${ }^{18}$ They claimed that their data supported the need to re-evaluate the role of palivizumab prophylaxis in infants born at a GA of $\geq 29$ weeks, but a significant difference in the prevalence of RSV infection-based hospitalisation was not observed during three consecutive RSV seasons from 2014 to 2017.

Debate as to which patients should receive palivizumab and which individuals do not benefit from such prophylaxis is continuing. Agreement and implementation of the updated AAP-2014 guidance was mixed among the various paediatric specialists. ${ }^{19}$ However, the consensus conference statement by Pignotti et al, ${ }^{20}$ which encompassed expert opinion and available evidence, suggests that palivizumab prophylaxis is not recommended for infants of GA $\geq 29$ weeks unless there is comorbidity. Nevertheless, the current Italian Guidelines recommend palivizumab prophylaxis for infants of GA 29-35 weeks and a chronological age $\leq 6$ months at the beginning of the epidemic season. ${ }^{21}$ 
These discrepancies, whereby palivizumab produces optimal benefit according to different sources of information (eg, SPC, national/international guidelines), places, prescribers and patients in a very difficult position. ${ }^{22}$ Hence, follow-up of a new decision on palivizumab prophylaxis that may have an impact on the neonatal health outcomes is crucial.

Several limitations of our study must be mentioned. Our analysis is based on data from one single Italian central region and may not reflect hospitalisation trends seen in other geographical area. However, to the best of our knowledge, this is the largest European study evaluating the impact of guidance on palivizumab based on population representing 10\% of Italian infants. We were not able to have laboratory confirmation of RSV diagnosis but our results are similar to other study capable to have also these data. ${ }^{12}$ This is an ecological study and cannot account for potential change of individual characteristics associated with neonatal outcomes but allowed to perform a prompt analysis of the impact of recent regulatory decision on the neonatal health outcomes of the target population. Our data are consistent with recent cohort study, ${ }^{14}$ where no differences were found in the rate of RSV hospitalisation in treated versus untreated with palivizumab.

\section{CONCLUSIONS}

We showed that implementation of NHS reimbursement criteria from 2016 for palivizumab was not associated, in a large central region of Italy, with an increased prevalence of RSV infection-based hospitalisation for infants aged $<2$ years. Regulatory decisions and updated guidelines followed by appropriate monitoring should represent the 'gold standard' for decision makers to understand the impact of such decisions on health outcomes and costs. We showed that pharmacoepidemiology can serve as a bridge between medical research and clinical practice.

Acknowledgements The authors would like to thank Tommaso Salvitti for support in the initial phase of data extraction.

Contributors VB and AA conceptualised and designed the study, performed the data analysis, drafted the manuscript. FT, LP and MD contributed to study design and reviewed the manuscript. Only public employees of the regional health authorities were involved in conceiving, planning and conducting the study.

Competing interests None declared.

Patient consent Not required.

Provenance and peer review Not commissioned; externally peer reviewed.

Open access This is an open access article distributed in accordance with the Creative Commons Attribution Non Commercial (CC BY-NC 4.0) license, which permits others to distribute, remix, adapt, build upon this work non-commercially, and license their derivative works on different terms, provided the original work is properly cited, appropriate credit is given, any changes made indicated, and the use is non-commercial. See: http://creativecommons.org/licenses/by-nc/4.0/.

\section{REFERENCES}

1 Bont L, Checchia PA, Fauroux B, et al. Defining the epidemiology and burden of severe respiratory syncytial virus infection among infants and children in western countries. Infect Dis Ther 2016:5:271-98.
2 Frassanito A, Nicolai A, Nenna R, et al. Acute viral respiratory tract infections and wheezing at follow-up in children. Eur Respir J 2015;46(Suppl 59):PA1319.

3 Ferrara M, Cangiano G, Papasso S, et al. Bronchiolitis: a 9 years epidemic seasons analysis. Eur Respir J 2014;44(Suppl 58):P1258.

4 Zuccotti G, Dilillo D, Zappa A, et al. Epidemiological and clinical features of respiratory viral infections in hospitalized children during the circulation of influenza virus A(H1N1) 2009. Influenza Other Respir Viruses 2011;5:e528-34.

5 Corsello G, Di Carlo P, Salsa L, et al. Respiratory syncytial virus infection in a Sicilian pediatric population: risk factors, epidemiology, and severity. Allergy Asthma Proc 2008:29:205-10

6 Medici MC, Arcangeletti MC, Rossi GA, et al. Four year incidence of respiratory syncytial virus infection in infants and young children referred to emergency departments for lower respiratory tract diseases in Italy: the "Osservatorio VRS" Study (2000-2004). New Microbiol 2006;29:35-43.

7 Lanari M, Giovannini M, Giuffré L, et al. Prevalence of respiratory syncytial virus infection in Italian infants hospitalized for acute lower respiratory tract infections, and association between respiratory syncytial virus infection risk factors and disease severity. Pediatr Pulmonol 2002:33:458-65.

8 Hall CB, Weinberg GA, Blumkin AK, et al. Respiratory syncytial virus-associated hospitalizations among children less than 24 months of age. Pediatrics 2013;132.

9 IMpact-RSV Study Group. Palivizumab, a humanized respiratory syncytial virus monoclonal antibody, reduces hospitalization from respiratory syncytial virus infection in high-risk infants. The IMpact-RSV Study Group. Pediatrics 1998;102:531-7.

10 American Academy of PediatricsCommittee on Infectious Diseases, American Academy of Pediatrics Bronchiolitis Guidelines Committee. Updated guidance for palivizumab prophylaxis among infants and young children at increased risk of hospitalization for respiratory syncytial virus infection. Pediatrics 2014;134:134 e620-e638.

11 Robinson JL, Le Saux N. Canadian Paediatric Society, Infectious Diseases and Immunization Committee. Preventing hospitalizations for respiratory syncytial virus infection. Paediatr Child Health 2015:20:321-6.

12 Grindeland CJ, Mauriello CT, Leedahl DD, et al. Association Between Updated Guideline-Based Palivizumab Administration and Hospitalizations for Respiratory Syncytial Virus Infections. Pediatr Infect Dis I 2016;35:728-32.

13 Rajah B, Sánchez PJ, Garcia-Maurino C, et al. Impact of the Updated Guidance for Palivizumab Prophylaxis against Respiratory Syncytial Virus Infection: A Single Center Experience. J Pediatr 2017;181:183-8.

14 Farber HJ, Buckwold FJ, Lachman B, et al. Observed Effectiveness of Palivizumab for 29-36-Week Gestation Infants. Pediatrics 2016;138:e20160627.

15 Buckley BC, Roylance D, Mitchell MP, et al. Description of the outcomes of prior authorization of palivizumab for prevention of respiratory syncytial virus infection in a managed care organization. J Manag Care Pharm 2010;16:15-22.

16 Newby B, Sorokan T. Respiratory syncytial virus infection rates with limited use of Palivizumab for infants born at 29 to $31+6 / 7$ weeks gestational age. Can J Hosp Pharm 2017:70:13-18.

17 Resch B, Bramreiter VS, Kurath-Koller S, et al. Respiratory syncytial virus associated hospitalizations in preterm infants of 29 to 32 weeks gestational age using a risk score tool for palivizumab prophylaxis. Eur I Clin Microbiol Infect Dis 2017:36:1057-62.

18 Capizzi A, Silvestri M, Orsi A, et al. The impact of the recent AAP changes in palivizumab authorization on RSV-induced bronchiolitis severity and incidence. Ital 」 Pediatr 2017;43:71.

19 Friedman DM, Domachowske JB, Wong PC, et al. Perceived Risk of Severe Respiratory Syncytial Virus Disease and Immunoprophylaxis Use Among US Pediatric Specialists. Clin Pediatr 2016;55:724-37.

20 Pignotti MS, Carmela Leo M, Pugi A, et al. Consensus conference on the appropriateness of palivizumab prophylaxis in respiratory syncytial virus disease. Pediatr Pulmonol 2016;51:1088-96.

21 Bollani L, Baraldi E, Chirico G, et al. Revised recommendations concerning palivizumab prophylaxis for respiratory syncytial virus (RSV). Ital J Pediatr 2015;15:97.

22 Addis A, Marchetti F, Bonati M. Registrazione, rimborso e uso appropriato dei farmaci non sono sinonimi: il caso del palivizumab. Medico e Bambino 2016:35:507-12. 